\title{
The role of difluprednate ophthalmic emulsion in clinical practice
}

This article was published in the following Dove Press journal:

Clinical Ophthalmology

25 June 2009

Number of times this article has been viewed

\author{
Karim N Jamal',2 \\ David G Callanan' \\ 'Texas Retina Associates, Arlington, \\ Texas, USA $;{ }^{2}$ Current affiliation: \\ Retinal Consultants of Arizona, \\ Phoenix, Arizona, USA
}

Correspondence: David Callanan I00I North Waldrop Drive, Suite $5 \mathrm{I} 2$, Arlington, TX 760I2, USA

$\mathrm{Tel}+\mathrm{I}(8 \mathrm{I})$ 26I-9625

$\mathrm{Fax}+\mathrm{I}(817)$ 26I-9586

Email dcallanan@texasretina.com
Abstract: The mainstay in the treatment of ocular inflammation, either post-surgical or endogenous, is the use of steroids. While these agents effectively address inflammation, they are not without their risks, including ocular hypertension and acceleration of cataract formation. The most notorious culprits are the strong steroids, such as prednisolone acetate and betamethasone. This review aims to cover the biochemistry and drug development of difluprednate, a novel synthetic strong steroid emulsion. In vivo pharmacokinetics as well as ocular distribution and metabolism are discussed, followed by a comprehensive summary of phase I, II, and III clinical trials evaluating safety and efficacy in patients suffering from postoperative inflammation or anterior uveitis. The objective is to provide an increased familiarity with this newly approved medication as a welcome addition to the ophthalmologist's armamentarium.

Keywords: difluprednate, steroid, emulsion, ophthalmic surgery

\section{Introduction}

Surgical technique in all fields of ophthalmology has evolved considerably over the years, from the transition to clear corneal incisions by anterior segment surgeons to the adoption of small-gauge minimally invasive pars plana vitrectomies by vitreoretinal specialists. Despite such technical advances, however, surgical manipulation of anterior segment structures triggers the release of arachidonic acid from cell membranes, leading to the production of prostaglandins and leukotrienes. These inflammatory mediators, in turn, lead to cellular reaction and protein leakage. Although often selflimited, untreated inflammation can lead to complications such as pain/discomfort, photophobia, corneal edema, synechiae, glaucoma, and cystoid macular edema. ${ }^{1,2}$

In the immediate postoperative period, topical corticosteroids are employed to suppress the production of inflammatory mediators, offering local treatment without the risk of systemic adverse effects. By inhibiting the release of arachidonic acid from cell membrane phospholipids, corticosteroids prevent the formation of both leukotrienes and prostaglandins, disrupting the inflammatory cascade. ${ }^{3}$ These agents are continued until the anterior chamber $(\mathrm{AC})$ reaction has resolved and the blood-aqueous barrier has been reestablished. ${ }^{4}$

Just as ophthalmologists have enjoyed advances in surgical technique and technology, patient expectations of their results have grown proportionately. Currently, the most widely prescribed strong topical corticosteroid in the US is prednisolone acetate $1 \%$. While it controls inflammation effectively, it has not been shown to consistently address postoperative pain and discomfort in a large clinical trial. ${ }^{5}$ In June 
2008 difluprednate ophthalmic emulsion $0.05 \%$ (Durezol $^{\mathrm{TM}}$; Sirion Therapeutics, Tampa, FL) was approved by the US Food and Drug Administration (FDA) for the treatment of inflammation and pain associated with ocular surgery - the first strong ophthalmic steroid approved by the FDA since 1973. Difluprednate is the first ophthalmic steroid developed in the past 35 years with high potency, a favorable safety profile, and the ability to reduce postoperative pain.

\section{Review of pharmacology,} formulation, pharmacokinetics, and in vivo analyses of difluprednate Pharmacology and drug development

Difluprednate (difluoroprednisolone butyrate acetate, or DFBA) is a synthetic difluorinated prednisolone derivative (Figure 1). Originally developed for dermatologic applications, the molecule derives its potency from fluorination at the $\mathrm{C} 6$ and $\mathrm{C} 9$ positions. ${ }^{6}$ Its anti-inflammatory activity is further augmented by replacing the 17-hydroxyl group with butyrate, while its lipophilicity - and hence corneal penetration - is enhanced by substituting the 21-hydroxyl group with acetate. ${ }^{7}$ Given the paucity of strong ophthalmic steroids available, difluprednate was formulated as a topical ophthalmic preparation.

Developing new ophthalmic treatments, however, involves numerous challenges. Many drugs are poorly soluble in water, for example. While this difficulty can be overcome by using surfactants, organic solvents, or a vehicle with $\mathrm{pH}$ outside physiological range, ${ }^{8-11}$ such modifications can lead to ocular irritation and limit use in clinical practice.

Ophthalmic suspensions (such as prednisolone acetate) can have potential problems such as flocculation, caking, and poor redispersibility, all of which can lead to dosing errors during administration. ${ }^{12,13}$ Dose uniformity also depends on

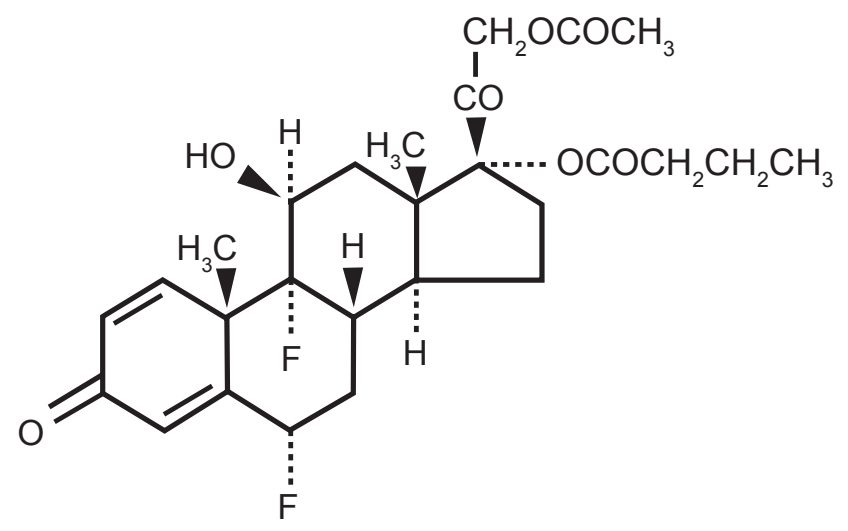

Figure I Difluprednate molecule. drug homogeneity, and suspensions have a large range of particle size, which can affect bioavailability. In addition, they must be shaken to suspend the medicine in the aqeous phase, otherwise the active drug settles at the bottom of the bottle thereby altering the dose delivered. Yet another obstacle is that particle variance precludes ophthalmic suspensions from undergoing a filtration technique of sterilization. ${ }^{7}$

One elegant solution to these problems is to create an oil-in-water lipid emulsion. This allows drugs that are poorly water soluble to dissolve in the oil phase (which does not have uniformity problems), undergo sterile filtration, and provide better ocular bioavailability. ${ }^{7}$ As mentioned above, lipid emulsions also require the addition of surfactants, and both high concentrations of these agents and the use of ionic varieties can lead to ocular toxicity. ${ }^{14}$ Selecting the appropriate oil and its concentration is also critical, since an emulsion's thermodynamic stability changes with the proportions of drug, surfactant, and oil; high oil concentrations, for example, can lead to blurred vision. ${ }^{15,16}$

The difluprednate emulsion formulation began by suspending DFBA in a variety of oils (castor, cottonseed, medium-chain fatty acid triglyceride, oleic, olive, peanut, and soybean). ${ }^{7}$ As castor oil showed the highest solubility, it was selected as the lipid phase. Next, various concentrations of polysorbate 80 , a nonionic emulsifying surfactant with a good safety profile for ophthalmic use, were added to determine the optimal proportion of drug, oil, and surfactant to avoid blurred vision and ocular surface toxicity. After several experiments, the final proportions were selected, with a resulting combination that was thermodynamically stable across a variety of storage conditions.

\section{Pharmacokinetics}

Once instilled, difluprednate emulsion is rapidly deacetylated in the aqueous humor to difluoroprednisolone butyrate (DFB), the drug's active metabolite, which has a similar corticosteroid activity profile. ${ }^{17}$ Endogenous tissue esterases then metabolize DFB to the inert metabolite hydroxyfluoroprednisolone butyrate (HFB), which limits systemic exposure to the active compound. ${ }^{18}$

Due to low drug concentrations and the possibility of contaminant proteins in aqueous humor, Yasueda et al ${ }^{19}$ used a combination of semi-micro high-performance liquid chromatography (HPLC) and column switching to detect in vivo ocular absorption of DFBA in a rabbit model. Using various concentrations of DFBA emulsion, the group found that DFBA could not be detected in aqueous humor samples at any concentration, whereas DFB was readily detected, 
indicating that the DFBA acetyl group was hydrolyzed quickly. They also reported that further DFB hydrolysis does not occur within the first hour after drop instillation.

In comparing the in vivo penetration of $0.05 \%$ difluprednate emulsion to suspension, Yamaguchi et $\mathrm{al}^{7}$ found that within 30 minutes of instillation the emulsion had a 7.4-foldhigher corneal DFB concentration than did the suspension. The emulsion also showed a 5.7- and 3.1-fold higher aqueous humor concentration at 1 and 3 hours, respectively, compared with suspension.

\section{Ocular distribution and metabolism}

To further evaluate the absorption, distribution, and metabolism of $0.05 \%$ DFBA emulsion, Tajika et a $1^{18}$ instilled a single drop of medication in both eyes of albino rabbits, and measured the concentrations of DFBA and DFB in ocular tissues and blood at several time points using the HPLC method. Because of the rapid deacetylation of DFBA to DFB, DFBA concentrations were undetectable in nearly all tissue samples, while DFB was found to accumulate throughout the anterior segment structures. DFB levels in the posterior segment and blood were undetectable at all time points.

In a separate study examining DFBA excretion, radiolabeled difluprednate was instilled in the right eyes of pigmented rabbits. ${ }^{18}$ After a single dose, radioactivity was detected in both anterior and posterior ocular structures, but not in the blood. At 24 hours postinstillation, $78.5 \%$ of radioactivity had been excreted, and $99.5 \%$ had been eliminated by 168 hours (7 days). Radioactivity levels did not increase markedly with an increased number of doses. Following 28 doses given over 7 days, concentrations distributed in the anterior segment were 1.5 times higher when compared to a single dose. There remained little accumulation in the blood, however, highlighting the rapid metabolism of DFB into inert products.

\section{Preclinical in vivo analyses}

Investigating the ocular and systemic safety of difluprednate emulsion, Sakaki et $\mathrm{al}^{20}$ compared the effects of difluprednate $0.01 \%$ to $0.05 \%$ in rabbits as well as difluprednate $0.05 \%$ to betamethasone sodium phosphate $0.1 \%$ in beagles. Betamethasone is a strong corticosteroid widely used in Europe, Canada, and Japan to treat ocular inflammation, and, on a molecular basis, is six times more potent than prednisolone. ${ }^{3}$

After four-times-daily (qid) dosing for 1 month, extensive evaluations were performed, including ophthalmic examination, hematology, blood chemistries, and organ histopathology. Physiological changes in the difluprednate group were slightly less severe than those seen in the betamethasone group, suggesting quick metabolism and therefore weak systemic exposure to DFBA. Overall, no significant ocular or systemic toxicities uncharacteristic of steroid use were noted in any group.

In a rabbit model, Inoue et $\mathrm{al}^{21}$ tested the effect of formulation and particle size on ocular bioavailability. Fifty microliters $(\mu \mathrm{L})$ of difluprednate $0.05 \%$ emulsion was instilled in the right eyes, while $50 \mu \mathrm{L}$ of the suspension form was administered to the left eyes. Using liquid chromatography tandem mass spectrometry, they found aqueous humor concentrations of difluprednate emulsion $0.05 \%$ to be higher at all time points following instillation than the suspension formulation. Further, the emulsion gave a 1.4-fold higher maximal concentration, providing $40 \%$ more bioavailability. Instilling emulsions with particle sizes between 90.3 and 129.3 nanometers $(\mathrm{nm})$ (standard particle size $110 \mathrm{~nm}$ ) gave similar aqueous concentrations at 1 hour post-instillation, indicating that difluprednate transfer was not affected by particle size.

A rabbit model was created both to assess the dose response of DFBA in postoperative inflammation and compare the effect of difluprednate $0.05 \%$ to betamethasone $0.1 \%{ }^{22}$ A laser flare cell meter was used to quantify AC protein leakage at several time points following surgical paracentesis and instillation of difluprednate $0.002 \%, 0.01 \%$, or $0.05 \%$; betamethasone $0.1 \%$, or normal saline. Difluprednate concentrations $\geq 0.01 \%$ showed a statistically significant inhibition of inflammation compared to saline, with the anti-inflammatory response proceeding in a dose-dependent manner $(P<0.01$ for difluprednate $0.01 \% ; P<0.001$ for difluprednate $0.05 \%$ ). Difluprednate $0.05 \%$ was also found to have an inhibitory effect equivalent to that of betamethasone $0.1 \%(P<0.001$ for each drug when compared to saline, no significant difference when compared to each other using the $t$ test).

To evaluate anti-inflammatory effects, diffuprednate $0.002 \%$, $0.01 \%$, and $0.05 \%$ were compared to betamethasone $0.1 \%$ in experimental melanin protein-induced uveitis in rats, bovine serum albumin-induced uveitis in rabbits, and endotoxininduced uveits in rats. ${ }^{23}$ Difluprednate suppressed uveitis in all three models in a dose-dependent manner, and difluprednate $0.05 \%$ showed statistically superior anti-inflammatory activity compared to betamethasone $0.1 \%$ ( $P<0.01$ in all models).

\section{Review of clinical studies and randomized trials} Early efficacy and safety studies

In 1999 a phase I study assessed the safety and tolerability of 3 concentrations of difluprednate ophthalmic emulsion given 
as a single instillation to otherwise healthy volunteers. ${ }^{24}$ In this randomized placebo-controlled single-masked trial, 18 patients were assigned in a 1:1:1 ratio to receive a single instillation of vehicle in one eye, and either difluprednate $0.002 \%, 0.01 \%$, or $0.05 \%$ in the fellow eye. Ocular examinations were conducted at several time points up to 24 hours post-installation, along with assessments of several ocular surface parameters and ERG testing. Subjective symptoms and tolerability were rated using a questionnaire. At the end of the 24-hour period, medical and ophthalmologic examinations were performed along with extensive blood work. All adverse effects (AEs) were mild, transient, and not necessarily related to drop instillation, demonstrating that a single installation of difluprednate at any of the concentrations tested was safe and well tolerated.

A second phase I study evaluated the safety, tolerability, and pharmacokinetics of 2 difluprednate concentrations administered qid for 7 days in healthy male volunteers. ${ }^{25}$ In this placebo-controlled, double-masked study, 12 subjects were assigned in a 1:1 ratio to receive 2 drops of placebo in 1 eye and 2 drops of either difluprednate $0.01 \%$ or $0.05 \%$ in the contralateral eye. Ophthalmic examinations, symptom questionnaires, physical examinations, and blood work were all conducted in a manner similar to that described above. Drug pharmacokinetics were determined by measuring serum drug concentration at several time points during the week. The results of this study demonstrated that topical difluprednate at either concentration was well tolerated and exhibited little systemic effect. No difluprednate was detected in any blood sample, with a detection limit of $50 \mathrm{ng} / \mathrm{mL}$. Once again, AEs were mild in intensity and transient in nature. There were three instances of elevated intraocular pressure (IOP), but none exceeded the normal range.

Next, a phase 2A study assessed the efficacy and safety of difluprednate emulsion $0.002 \%$ or $0.05 \%$ administered qid for 7 days following cataract surgery. ${ }^{26}$ Because of strict eligibility requirements, this randomized, double-masked, parallel-group comparative study enrolled only 6 patients, precluding a direct comparison of the two concentrations. Efficacy was determined by quantifying AC cells and flare, while safety was assessed by ophthalmic examination, a subjective evaluation, and blood work. The results of this study indicated that both concentrations of difluprednate were effective and well tolerated for the treatment of postoperative inflammation. All AEs were mild or moderate in severity and transient in nature.

A multicenter randomized parallel-group active-control phase 2B clinical study compared the efficacy and safety of difluprednate $0.05 \%$ to betamethasone $0.1 \%$ for the treatment of postoperative inflammation. ${ }^{27}$ Twenty-four patients with AC cell scores $\geq 2$ (10 to 20 cells per high-powered field), were randomized to receive either difluprednate $0.05 \%$ or betamethasone $0.1 \%$ qid for 14 days. Efficacy and safety were measured in a manner similar to that described above. There were no statistically significant differences between the treatment groups in mean AC cell count, mean AC flare, or mean total symptoms on days 3,7 , or 14 . In both arms, most measures were significantly improved from baseline on days 7 and 14. There were no serious AEs during the study period, and only one patient in the difluprednate group experienced mild IOP elevation, which resolved following treatment with a topical antiglaucoma medication. This study showed that treatment with difluprednate $0.05 \%$ was at least as effective as betamethasone $0.1 \%$ in reducing postoperative inflammation, and that its safety profile was acceptable.

\section{Larger randomized clinical and comparative trials} Postoperative inflammation

A phase 3 multicenter randomized double-masked parallelgroup comparative noninferiority trial conducted in Japan assessed the safety and efficacy of $0.05 \%$ difluprednate to $0.1 \%$ betamethasone for the treatment of postoperative inflammation following cataract or vitreous surgery. ${ }^{28}$ One hundred eighty-two patients with AC cell scores of 2 or higher (10+ cells per high-powered field) were randomized to receive one of the medications qid for 14 days. The primary endpoint used to compare the two treatments was change from baseline in AC cell score on day 14. Secondary endpoints included changes in cell score at interim time points, as well as changes from baseline in AC flare, total signs, and total symptom score.

At the completion of the study period, postoperative inflammation was similarly reduced in both groups, verifying the study's noninferiority hypothesis $(P<0.01)$. Analysis of secondary endpoints revealed no differences between difluprednate and betamethasone in either AC flare or total sign score - except for day 7 when the difluprednate arm showed a statistically significant improvement in total sign score, including hyperemia, chemosis, and keratic precipitates. The difluprednate group also showed a statistically significant improvement compared to the betamethasone group in subjective symptoms, including pain, photophobia, foreign body sensation, and blurred vision at all time points after the initiation of therapy. A few patients in each group experienced 
elevated IOP, all of which resolved spontaneously or with the addition of a topical agent. This study verified that difluprednate was at least as effective as betamethasone in treating postoperative inflammation, and that it had a favorable safety profile.

Two identical Phase 3 multicenter randomized repeateddose double-masked parallel-group placebo-controlled trials were conducted at 26 sites in the United States to assess the safety and efficacy of difluprednate emulsion. Four hundred thirty-eight patients with AC cell scores of grade 2 or higher ( $>10$ cells per high-powered field) following ocular surgery ${ }^{29}$ were randomized to one of four treatment arms: difluprednate twice daily (bid) $(\mathrm{n}=111)$, difluprednate qid $(n=107)$, or placebo dosed two or four times daily $(n=110$ each). Patients were instructed to use the medication at the assigned frequency; if their inflammation had responded satisfactorily at day 15 , they entered a tapering schedule. Outcome measures were $\mathrm{AC}$ cell grade, $\mathrm{AC}$ flare score, and a quantitative evaluation of pain, discomfort, and photophobia.

As early as day 3 there was a mean decrease in AC cell grade, from an initial baseline of approximately 2.4 , to 1.0 in the difluprednate bid group and 0.8 in the difluprednate qid group, compared to 0.4 in the placebo groups. This correlated with an $87 \%$ reduction in $\mathrm{AC}$ cell count in the difluprednate groups versus only a $30 \%$ reduction in the placebo groups. These responses were sustained throughout the study. The proportion of patients achieving an AC cell grade of 0 (defined as $\leq 1$ cell per high-powered field) rose over time, reaching $77 \%$ in the difluprednate twice-daily group and $81 \%$ in the difluprednate four-times-daily group, compared to $25 \%$ in the placebo group by day $29(P<0.0001)$.

The proportion of patients with a clinical response (defined as $\leq 5 \mathrm{AC}$ cells and no flare) was noted as early as day 3 , with significant differences observed by day 8 . At 1 week after surgery, $46.4 \%$ of patients in the difluprednate bid (twice daily dosing) group and $42.1 \%$ in the difluprednate qid group had achieved a clinical response (Figure 2), compared with $18.9 \%$ in the placebo group $(P<0.0001)$. This trend continued to increase, and by day $29,79.1 \%$ and $82.2 \%$ of patients in the difluprednate bid and qid groups, respectively, had a clinical response versus $39.4 \%$ in the placebo group $(P<0.0001)$.

Difluprednate also reduced pain as early as day 3 , with $38.2 \%$ of the bid patients $(P=0.0125)$ and $45.3 \%$ of the four-times-daily patients $(P<0.0001)$ claiming to be pain and discomfort free, versus $24.8 \%$ of patients in the placebo group. On day 3 , patients randomized to difluprednate had a substantial reduction in photophobia from baseline ( $P=0.0041$ in the bid group, $P<0.0001$ in the qid group),

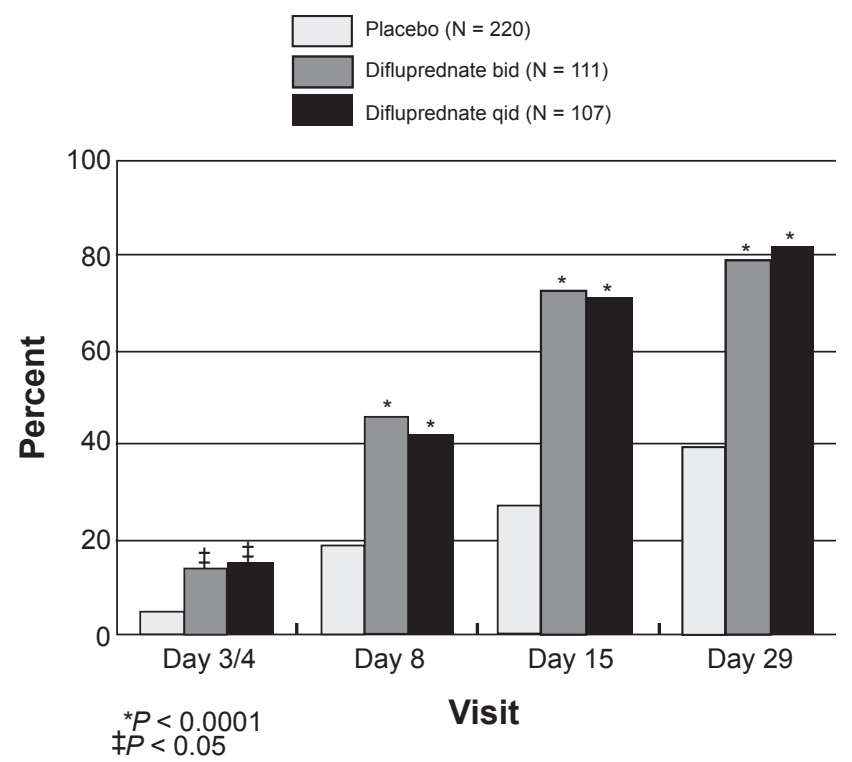

Figure 2 Phase 3 postoperative trial: Percent of subjects with clinical response (anterior chamber cell count $\leq 5$ and flare grade 0 ).

while scores for placebo-treated patients worsened. The trends established by the difluprednate groups continued throughout the study.

The mean IOP remained within the normal range in all treatment groups, with only 3 patients in each difluprednate group and 2 patients in the placebo groups experiencing a clinically significant IOP rise (defined as $\geq 10 \mathrm{~mm} \mathrm{Hg}$ from baseline and $\geq 21 \mathrm{~mm} \mathrm{Hg}$ overall). Elevated IOP was effectively controlled with topical medication. No serious ocular AEs were reported in any treatment group, while systemic events were typical for the geriatric study population, giving no indication that difluprednate had caused target organ toxicity.

This multicenter randomized trial once again demonstated the safety and efficacy of difluprednate emulsion. More important, however, was the finding that the signs and symptoms seen following ocular surgery were effectively treated with twice-daily dosing. Less frequent dosing may engender better patient compliance and reduce total steroid exposure.

\section{Anterior uveitis}

In a phase 3 noninferiority study conducted in Japan, difluprednate $0.05 \%$ was compared to betamethasone $0.1 \%$ in patients with endogenous anterior uveitis. ${ }^{30}$ One hundred thirty-six patients with AC cell scores of 2 to 3 (10 to 49 cells per high-powered field) were randomized 1:1 to receive either drug qid for 14 days. Of the 136 patients, 127 completed the trial and were included in the efficacy analyses. 
The primary endpoint was change in AC cell score from baseline to day 14. AC flare and ocular signs and symptoms were also evaluated.

At day 14 improvement in AC cell scores were comparable between both treatments, corroborating the study's noninferiority hypothesis. However, difluprednate produced a substantially more rapid improvement: By day 7 more patients in the difluprednate group had AC cell scores of 1 or lower $(P=0.0298)$ (Figure 3$)$. Similar findings were noted when examining secondary efficacy measures such as AC flare score $(P<0.05)$ and total sign score $(P=0.0355)$. The incidence of elevated IOP was equal between the two groups, and resolved with or without medical treatment. None of the patients in the difluprednate arm withdrew from the study due to symptom aggravation, compared to 3 patients in the betamethasone group.

The safety and efficiacy of difluprednate ophthalmic emulsion $0.05 \%$ was further evaluated in an open-label phase 3 trial of 19 patients with severe refractory endogenous anterior uveitis ( $\geq 50$ cells per high-powered field in the anterior chamber). ${ }^{31}$ These patients had not responded to previous treatment with betamethasone $0.1 \%$, even when given at a frequency greater than the recommended qid dosing specified on its label; most had been dosed between 8 and 12 times per day. The primary efficacy measure was the change in AC cell score from baseline to day 14. AC flare score as well as total signs and total symptoms were also quantified at several time points.

Difluprednate $0.05 \%$ dosed qid demonstrated a statistically significant improvement in mean AC cell score from baseline $[4.0 \pm 0.0$ (mean \pm standard deviation) $]$ to day 14 $(1.3 \pm 0.8)(P<0.0001)$ in the 18 patients who completed the study (Figure 4). Significant improvements from baseline were also observed on day $3(P<0.0001)$ and day 7

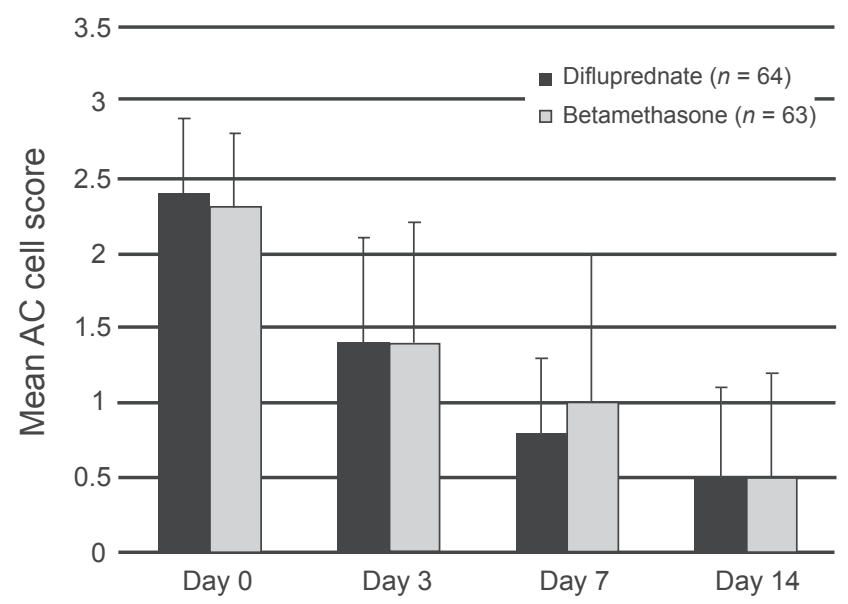

Figure 3 Phase 3 uveitis trial. Mean anterior chamber (AC) cell score over time.

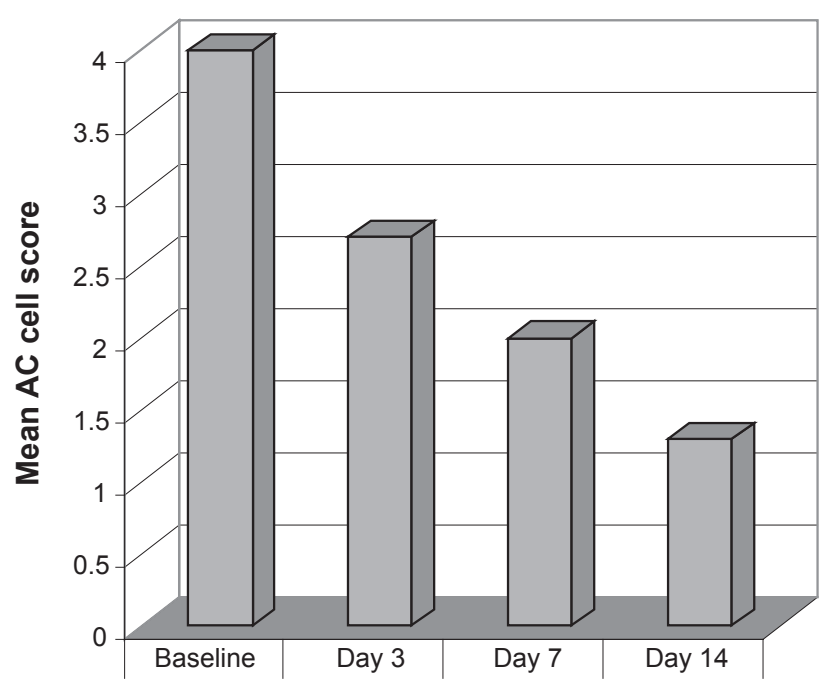

Figure 4 Phase 3 open-label refractory uveitis trial. Anterior chamber (AC) cell score mean change from baseline.

$(P<0.0001)$. In 13 of the 18 patients $(72.2 \%)$, the AC cell score had improved to 1 or less by day 14, with 2 patients reaching 0 (Figure 5). Significant improvement from baseline in AC flare, total sign and total symptom scores were noted at days 3,7 , and 14 ( $P \leq 0.0002$ at all measures). In all patients, difluprednate was well tolerated, with only 2 participants experiencing IOP elevation, both controlled with topical beta blocker.

Most recently, a multicenter randomized double-masked trial compared the efficacy and safety of difluprednate $0.05 \%$ dosed qid to prednisolone acetate $1 \%$ dosed 8 times daily. ${ }^{32}$ Ninety patients with endogenous anterior uveitis were randomized into 2 treatment arms to receive study medications at the above doses for 14 days, followed by 2 weeks of tapering at half the dose, and then 2 weeks of follow-up. The primary endpoint was the change in AC cell score from baseline.

At day 14, the mean cell grade reduction was 2.1 in the difluprednate arm, compared to 1.9 in the prednisolone acetate group, confirming the noninferiority of difluprednate qid to prednisolone 8 times daily (Figure 6). Several additional efficacy endpoints were achieved, but because this was a noninferiority study, the trial was not powered to reveal statistically significant differences between the two treatments. A greater proportion of patients receiving difluprednate had an AC cell score of $0(69 \%)$ than those using prednisolone (62\%). Difluprednate also demonstrated an advantage in pain relief from baseline (Figure 7), a greater mean reduction in total symptom score, and a greater reduction in total sign score. 


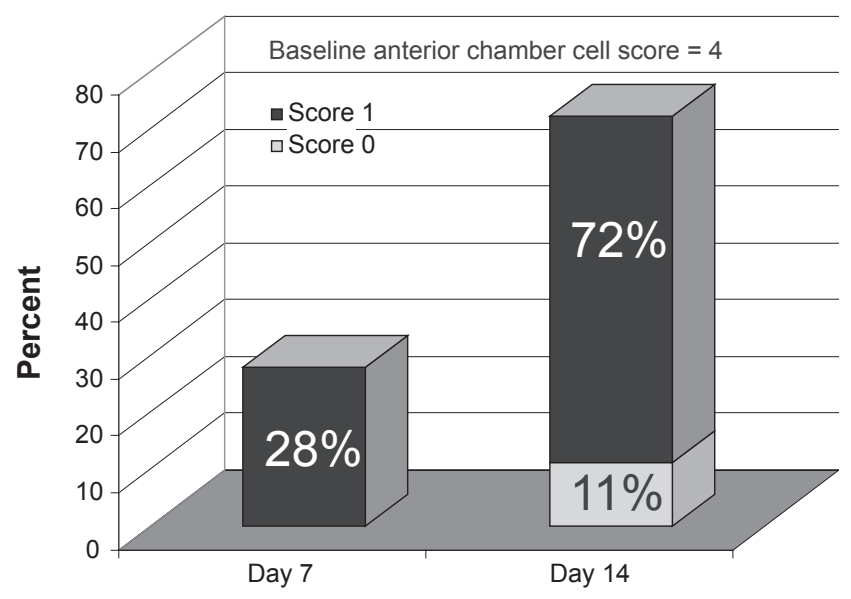

Figure 5 Phase 3 open-label refractory uveitis trial. Percent of patients with anterior chamber $(A C)$ cell score of 0 or $\mathrm{I}$.

No patients in the difluprednate arm withdrew from the study, while $12.5 \%$ of patients using prednisolone were withdrawn for lack of efficacy or adverse effects $(P=0.01)$. Three patients in the difluprednate treatment group and 2 in the prednisolone group experienced increased IOP. The findings of this trial, along with the consistent numerical advantage of difluprednate across all endpoints, demonstrate its potency and efficacy in treating anterior uveitis.

\section{Safety and tolerability}

It has long been known that topical corticosteroids especially strong steroids such as dexamethasone - can lead to an IOP increase. Ocular hypertension often occurs as early as 1 week after repeat dosing, ${ }^{33}$ an effect that can be much more pronounced in glaucomatous eyes. ${ }^{34}$ IOP elevation over long periods is associated with optic nerve damage, leading to visual field defects and possible reduction in acuity. Other well documented effects of topical steroids include formation of posterior subcapsular cataracts and a predisposition to secondary ocular infections.

Extensive clinical testing has demonstrated that difluprednate $0.05 \%$ emulsion causes an elevation in IOP in a small minority of patients. This increase resolved in all patients after stopping the medication or with topical pressure-lowering drops. Compared with betamethasone dosed at equal frequency, the incidence of IOP elevation was essentially equal between the two groups, ${ }^{27,28,30}$ indicating an acceptable safety level.

Benzalkonium chloride (BAK) is a quaternary ammonium detergent ${ }^{35,36}$ used as a preservative in many ophthalmic products. BAK is known to break down cell walls by emulsifying membrane lipids, ${ }^{37}$ which disrupts the tear film causing immunoallergic reactions, ${ }^{38}$ and creates direct toxicity to corneal and conjunctival epithelial cells. Difluprednate ophthalmic emulsion does not contain BAK, and instead uses sorbic acid as a preservative. Sorbic acid causes little damage and irritation to the ocular surface and is recommended for use in sensitive eyes. ${ }^{39}$

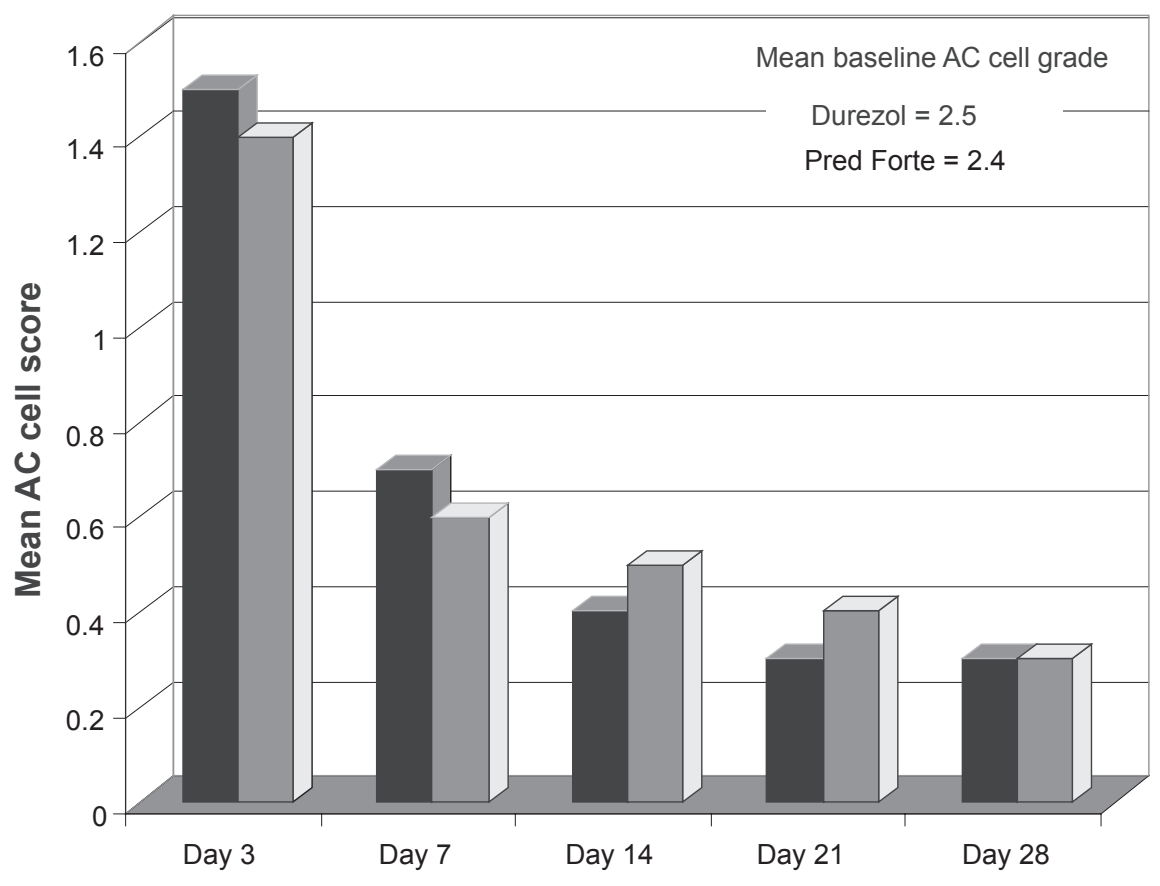

Figure 6 Sirion phase 3 uveitis trial: anterior chamber (AC) cell score change over time. 


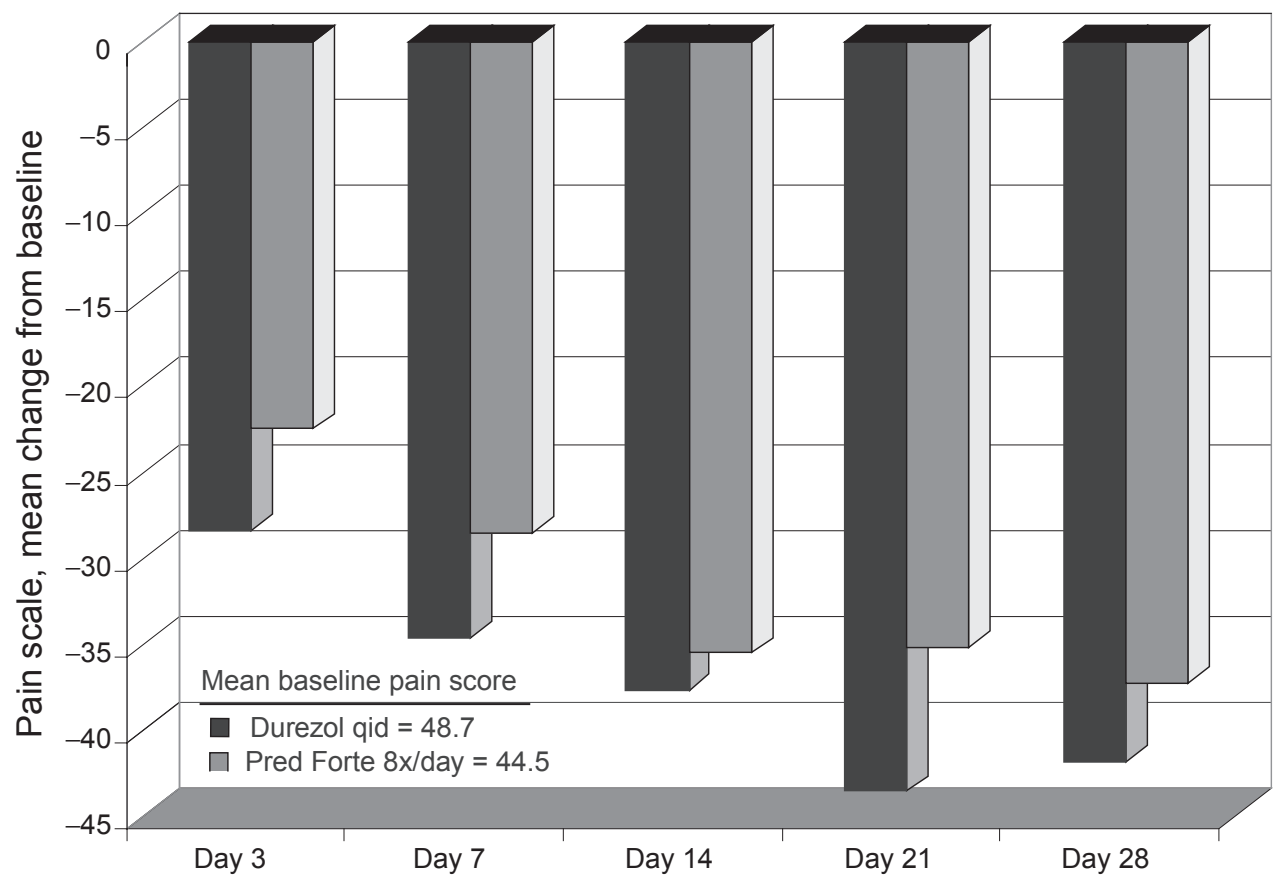

Figure 7 Sirion phase 3 uveitis trial: pain score - mean change from baseline.

Data from phase 1 trials of single- and repeated-dose difluprednate show that ocular adverse events were mild and transient. Safety data from other studies indicate difluprednate is well tolerated following ocular surgery and in the treatment of anterior uveitis.

\section{Role in clinical practice}

Patient expectations of surgical results and postoperative comfort have advanced with evolving surgical techniques and instrumentation. Despite this, no strong steroids have been approved by the FDA for the treatment of ocular inflammation since 1973. Technological improvments in pharmaceutical development now permit the creation of potent topical steroids with better bioavailability and rapid local metabolism, both of which minimize systemic exposure. Although weaker steroids and nonsteroidal antiinflammatory drugs may have a better safety profile, many patients will require the strongest available steroid to control their inflammation.

In the United States, postsurgical inflammation is treated prophylactically with prednisolone acetate; in other countries, betamethasone sodium phosphate is the standard of care. If left untreated, chronic inflammation can lead to further complications, such as pain and discomfort, cystoid macular edema, elevated IOP, synechiae, or keratopathy. ${ }^{40}$ Glucocorticoid gene activation assays have shown betamethasone to be intrinsically stronger than prednisolone,${ }^{41}$ making betamethasone a reference drug when evaluating new corticosteroids for the treatment of ocular inflammation. ${ }^{42}$

In an animal model, difluprednate was found to have higher anti-inflammatory activity than betamethasone. ${ }^{23}$ Clinical trials have shown that difluprednate is at least comparable to betamethasone in treating postoperative inflammation.$^{28}$ Further, unlike current-generation topical steroids, difluprednate is the first topical steroid indicated for the treatment of both postoperative inflammation and pain.

Difluprednate has also shown promising results in the treatment of anterior uveitis. Uveitis is the third leading cause of blindness in the United States, and is estimated to be responsible for 30,000 new cases of legal blindness each year. ${ }^{43}$ Uveitis may cause up to $15 \%$ of all cases of blindness. ${ }^{44}$

In patients with moderate anterior uveitis, difluprednate yielded a significantly faster improvement than betamethasone, suggesting that it has a quicker and more potent anti-inflammatory effect. ${ }^{30}$ Difluprednate has also been shown to reduce inflammation in cases of severe recalcitrant uveitis that had failed to respond to previous betamethasone therapy, ${ }^{31}$ and has also produced similar anti-inflammatory results compared with prednisolone dosed at twice the frequency. ${ }^{32}$

The potency and limited systemic absorption of difluprednate make it an attractive option when treating chronic diseases such as uveitis. In March 2009, the FDA accepted 
for review a New Drug Application for difluprednate to treat endogenous anterior uveitis filed by Sirion Therapeutics.

Although currently approved for qid dosing, data analysis revealed that difluprednate bid treated postoperative inflammation and discomfort effectively, even outperforming the qid group at some time points. From the patient's perspective, less frequent dosing may lead to better compliance. ${ }^{45}$ For the physician, twice-daily dosing allows flexibility when creating therapeutic regimens. The lower dosing reduces the risks of steroid exposure and permits the confident creation of patient-specific drop schedules, taking into account past medical and ocular histories, such as glaucoma.

In summary, difluprednate $0.05 \%$ ophthalmic emulsion is a potent new topical steroid that exhibits enhanced penetration, better bioavailability, rapid local metabolism, and strong efficacy with low incidence of adverse effects. It is the first strong ophthalmic steroid to be developed in over 30 years, and is effective in treating both postoperative inflammation and anterior uveitis.

\section{Disclosures}

The authors have no financial involvement, financial interests, or financial conflicts with Sirion Therapeutics or with difluprednate ophthalmic emulsion.

\section{References}

1. Apple DJ, Solomon KD, Tetz MR, et al. Posterior capsule opacification. Surv Ophthalmol. 1992;37:73-116.

2. Miyake K, Maekubo K, Miyake Y, et al. Pupillary fibrin membrane; a frequent early complication after posterior chamber lens implantation in Japan. Ophthalmol. 1989;96:1228-1233.

3. Schimmer BP, Parker KL. Adrenocorticotropic hormone; adrenocortical steroids and their synthetic analogs; inhibitors of the synthesis and actions of adrenocortical hormones. In: Hardman JG, Limbird LE, Gilman AG, editors. Goodman \& Gilman's The Pharmacological Basis of Therapeutics, 10th Edition. New York: McGraw-Hill; 2001. p. 1649-1677.

4. Sanders DR, Kraff M. Steroidal and nonsteroidal anti-inflammatory agents. Effect on postsurgical inflammation and blood-aqueous humor barrier breakdown. Arch Ophthalmol. 1984;102(10):1453-1456.

5. Korenfeld M. Difluprednate: changing the landscape of ocular pharmacology. Expert Rev Ophthamol. 2008;3(6):619-625.

6. Bikowski J. The position not the presence of the halogen in corticosteroids influences potency and side effects. J Drugs Dermatol. 2006;5(2):125-130.

7. Yamaguchi M, Yasueda S, Isowaki A, et al. Formulation of an ophthalmic lipid emulsion containing an anti-inflammatory steroidal drug, difluprednate. Int J Pharmaceutics. 2005;301:121-128.

8. Sweetana S, Akers MJ. Solubility principles and practices for parenteral drug dosage form development. J Pharm Sci Technol. 1996;50: 330-342.

9. Rajagopalan N, Dicken CM, Ravin LJ, et al. A study of the solubility of amphotericin B in nonaqueous solvent systems. J Parenter Sci Technol. 1988;42:97-102.

10. Loftsson T, Petersen DS. Cyclodextrin solubilization of ETH-615, a zwitterionic drug. Drug Dev Ind Pharm. 1998;24:365-370.

11. Alvarez-Nunez FA, Yalkowsky SH. Solubilization of diazepam. J Pharm Sci Technol. 1998;52:33-36.
12. Deicke A, Suverkrup R. Dose uniformity and redispersibility of pharmaceutical suspensions. I: quantification and mechanical modeling of human shaking behaviour. Eur J Pharm Biopharm. 1999;48: $225-232$.

13. Diestelhorst M, Kwon KA, Suverkrup R. Dose uniformity of ophthalmic suspensions. J Cataract Refract Surg. 1998;24:672-677.

14. Vandamme T. Microemulsions as ocular drug delivery systems: recent developments and future challenges. Prog Retin Eye Res. 2002;21: 15-34.

15. Koycha M, Rochat MH, Verain A. Physicochemical stability of emulsions: application to mixtures for parenteral nutrition. J Pharm Belg. 1988;43:413-424.

16. Tamilvanan S, Benita S. The potential of lipid emulsion for ocular drug delivery of lipophilic drugs. Eur J Pharm Biopharm. 2004;58:357-368.

17. Fujino A, Ohtu S, Shibata K, et al. Studies on the metabolic fate of difluprednate (DFBA). 3. Metabolism in rats and rabbits after subcutaneous administration. Pharmacometrics. 1985;29:713-723.

18. Tajika T, Shirasaki Y, Kimura M, et al. Ocular distribution and metabolism after instillation of difluprednate ophthalmic emulsion in rabbits. The 2007 Annual Meeting of the Association for Research in Vision and Ophthalmology (ARVO). Poster B744, Program 2654. Fort Lauderdale, FL, USA, 6-10 May 2007.

19. Yasueda S, Kimura M, Ohtori A, et al. Analysis of an anti-inflammatory steroidal drug, difluprednate, in aqueous humor by combination of semimicro HPLC and column switching method. J Pharm Biomed Anal. 2003;30:1735-1742.

20. Sakaki H, Mito C, Nemoto S, et al. Preclinical toxicity of difluprednate ophthalmic emulsion. The 2007 Annual Meeting of the Association for Research in Vision and Ophthalmology (ARVO). Poster B743, Program 2653. Fort Lauderdale, FL, USA, 6-10 May 2007.

21. Inoue J, Yamaguchi M, Sakaki H, et al. Preclinical pharmacokinetics of difluprednate ophthalmic emulsion. The 2007 Annual Meeting of the Association for Research in Vision and Ophthalmology (ARVO). Poster B741, Program 2651. Fort Lauderdale, FL, USA, 6-10 May 2007.

22. Kida T, Takahaski H, Tsuzuki M, et al. Difluprednate emulsion inhibits postoperative inflammation in rabbit paracentesis model. The 2007 Annual Meeting of the Association for Research in Vision and Ophthalmology (ARVO). Poster B745, Program 2655. Fort Lauderdale, FL, USA, 6-10 May 2007.

23. Okumura A, Tsuzuki M, Wada T, et al. Efficacy of difluprednate ophthalmic emulsion in preclinical studies of uveitisThe 2007 Annual Meeting of the Association for Research in Vision and Ophthalmology (ARVO). Poster B742, Program 2652. Fort Lauderdale, FL, USA, 6-10 May 2007.

24. Senju Pharmaceutical Co., Ltd., Osaka, Japan. Phase I clinical study of difluprednate: single instillation. Mar 1999. Data on file.

25. Senju Pharmaceutical Co., Ltd., Osaka, Japan. Phase I clinical study of difluprednate: repeated instillation. Mar 1999. Data on file.

26. Senju Pharmaceutical Co., Ltd., Osaka, Japan. Phase IIa clinical study of difluprednate $0.002 \%$ and $0.05 \%$ in the treatment of postoperative inflammation. Feb 2002. Data on file.

27. Senju Pharmaceutical Co., Ltd., Osaka, Japan. Phase IIb clinical study of difluprednate $0.05 \%$ vs betamethasone $0.1 \%$ in the treatment of postoperative inflammation. Apr 2004. Data on file.

28. Ohji M, Tano Y, Hida T, et al. Efficacy and safety results of a phase III study of difluprednate ophthalmic emulsion $0.05 \%$ in postoperative inflammation. The 2007 Annual Meeting of the Association for Research in Vision and Ophthalmology (ARVO). Poster B807, Program 3903. Fort Lauderdale, FL, USA, 6-10 May 2007.

29. Korenfeld MS, Silverstein SM, Cooke DL, et al. Difluprednate ophthalmic emulsion $0.05 \%$ for postoperative inflammation and pain. J Cataract Refract Surg. 2009;35:26-34.

30. Ohno S, Mochizuki M, Usui M, et al. A phase III noninferiority study of difluprednate ophthalmic emulsion $0.05 \%$ in the treatment of anterior uveitis. The 2007 Annual Meeting of the Association for Research in Vision and Ophthalmology (ARVO). Poster B808, Program 3904. Fort Lauderdale, FL, USA, 6-10 May 2007. 
31. Mochizuki M, Ohno S, Usui M, et al. A phase III open-label clinical study of difluprednate ophthalmic emulsion $0.05 \%$ in the treatment of severe refractory anterior uveitis. The 2007 Annual Meeting of the Association for Research in Vision and Ophthalmology (ARVO). Poster B809, Program 3905. Fort Lauderdale, FL, USA, 6-10 May 2007.

32. Sirion Therapeutics, Inc., Tampa, FL. A phase III multicenter, randomized, double-masked study of the safety and efficacy of difluprednate $0.05 \%$ ophthalmic emulsion compared to prednisolone acetate $1 \%$ ophthalmic suspension in the treatment of endogenous anterior uveitis. Dec 2008. Data on file.

33. Armaly MF. Effect of corticosteroids on intraocular pressure and fluid dynamics. I. The effect of dexamethasone in the normal eye. Arch Ophthalmol. 1963;70:482-491.

34. Armaly MF. Effect of corticosteroids on intraocular pressure and fluid dynamics. II. The effect of dexamethasone in the glaucomatous eye. Arch Ophthalmol. 1963;70:492-499.

35. Baudouin C. Allergic reaction to topical eyedrops. Curr Opin Allergy Clin Immunol. 2005;5:459-463.

36. Price F. Do you welcome BAK? Weighing the toxicity risk. Optometric Management. 2004 Mar [accessed 2009 Apr 5]. Available from: http:// www.optometric.com/article.aspx?articleZ71020.

37. Dart J. Corneal toxicity: the epithelium and stroma in iatrogenic and factitious disease. Eye. 2003;17:886-892.

38. Yee RW. The effect of drop vehicle on the efficacy and side effects of topical glaucoma therapy: a review. Curr Opin Ophthalmol. 2007;18: 134-139.
39. Abelson MB, Washburn S. The downside of tear preservatives. Rev Ophthalmol. 2002;9:102-106.

40. Negi AK, Browning AC, Vernon SA. Single perioperative triamcinolone injection versus standard postoperative steroid drops after uneventful phacoemulsification surgery: randomized controlled trial. J Cataract Refract Surg. 2006;32(3):468-474.

41. Diederich S, Scholz T, Eigendorff E, et al. Pharmacodynamics and pharmacokinetics of synthetic mineralocorticoids and glucocorticoids: receptor transactivation and prereceptor metabolism by ${ }^{11}$ betahydroxysteroid-dehydrogenases. Horm Metab Res. 2004;36:423-429.

42. Dunne JA, Jacobs N, Morrison A, et al. Efficacy in anterior uveitis of two known steroids and topical tolmetin. Br J Ophthalmol. 1985; 69:120-125.

43. Statistics on Blindness and Blinding Diseases in the United States [web page]. The University of Washington Department of Ophthalmology; c1997-2004 [updated 2004 Jan 1; accessed 2009 Apr 5]. Available from: http://depts.washington.edu/ophthweb/statistics.html.

44. Frequently Asked Questions [web page]. American Uveitis Society; c2002 [accessed 2009 Apr 5]. Available at: http://www.uveitissociety. org/pages/faq.html.

45. Eisen SA, Miller DK, Woodward RS, et al. The effect of prescribed daily dose frequency on patient medication compliance. Arch Intern Med. 1990;150:1881-1884.
Clinical Ophthalmology

\section{Publish your work in this journal}

Clinical Ophthalmology is an international, peer-reviewed journal covering all subspecialties within ophthalmology. Key topics include: Optometry; Visual science; Pharmacology and drug therapy in eye diseases; Basic Sciences; Primary and Secondary eye care; Patient Safety and Quality of Care Improvements. This journal is indexed on

\section{Dovepress}

PubMed Central and CAS, and is the official journal of The Society of Clinical Ophthalmology (SCO). The manuscript management system is completely online and includes a very quick and fair peer-review system, which is all easy to use. Visit http://www.dovepress.com/ testimonials.php to read real quotes from published authors. 